\title{
Cardiac metastasis of squamous cell carcinoma of the thyroid gland with severe disseminated intravascular coagulation: A case report
}

\author{
TOMOYASU YOSHIHIRO ${ }^{1}$, KENJI TSUCHIHASHI ${ }^{1}$, HITOSHI KUSABA ${ }^{1}$, TORAHIKO NAKASHIMA ${ }^{2}$, \\ TEPPEI OBARA ${ }^{1}$, KENTA NIO ${ }^{1}$, KOTOE TAKAYOSHI ${ }^{1}$, HIROYUKI KODAMA ${ }^{1}$, NOBUHIRO TSURUTA ${ }^{1}$, \\ HIDEYUKI KIYOHARA ${ }^{2}$, KAORI ASAI ${ }^{3}$, EIJI HARADA ${ }^{4}$, KENJIRO KAMEZAKI ${ }^{1}$, TAKESHI ARITA ${ }^{1}$, \\ MASANOBU SATO ${ }^{5}$, HIDETAKA YAMAMOTO ${ }^{5}$, SHUJI ARITA $^{6}$, HIROSHI ARIYAMA ${ }^{1}$, \\ KEITA ODASHIRO $^{1}$, YOSHINAO ODA ${ }^{5}$, KOICHI AKASHI ${ }^{1}$ and EISHI BABA ${ }^{6}$ \\ Departments of ${ }^{1}$ Medicine and Biosystemic Science, ${ }^{2}$ Otorhinolaryngology and ${ }^{3}$ Clinical Radiology; \\ ${ }^{4}$ Research Institute for Diseases of the Chest; ${ }^{5}$ Department of Anatomic Pathology, Pathological Sciences, \\ Graduate School of Medical Sciences; ${ }^{6}$ Department of Comprehensive Clinical Oncology, \\ Faculty of Medical Sciences, Kyushu University, Fukuoka 812-8582, Japan
}

Received August 29, 2016; Accepted October 21, 2016

DOI: $10.3892 / \mathrm{mco} .2016 .1091$

\begin{abstract}
Distant metastasis of primary squamous cell carcinoma (SCC) of the thyroid gland is rare and, to the best of our knowledge, cardiac metastasis has not been reported to date. A 57-year-old man underwent surgery and adjuvant chemoradiotherapy for stage IVA SCC of the thyroid gland. After 3 months, the patient was admitted to the Kyushu University Hospital (Fukuoka, Japan) with subcutaneous hematomas of the left thigh and lower leg, and he was diagnosed with cardiac and mediastinal lymph node metastases of SCC of the thyroid gland with severe disseminated intravascular coagulation (DIC). Echocardiography revealed a mass, $52 \mathrm{~mm}$ in greatest diameter, protruding from the interventricular septum towards the right ventricle. Weekly administration of paclitaxel and concurrent irradiation of the cardiac and lymph node metastases were performed. Eighteen days after the initiation of chemoradiotherapy, the DIC and hematomas had significantly improved, and the cardiac metastasis was stable. However, 2 months after admission, the patient developed dyspnea and multiple nodular shadows appeared to be spreading in the subpleura of the lungs bilaterally, which were initially suspected to be pulmonary tumor embolisms. Prednisolone and subsequent administration
\end{abstract}

Correspondence to: Professor Eishi Baba, Department of Comprehensive Clinical Oncology, Faculty of Medical Sciences, Kyushu University, 3-1-1 Maidashi, Higashi-ku, Fukuoka 812-8582, Japan

E-mail: e-baba@c-oncology.med.kyushu-u.ac.jp

Key words: squamous cell carcinoma of the thyroid gland, cardiac metastasis, disseminated intravascular coagulation, chemoradiotherapy of lenvatinib were not effective and the patient succumbed to respiratory failure. Severe DIC caused by extremely rare cardiac metastasis of SCC of the thyroid gland was effectively controlled by chemoradiotherapy. However, intensive local control appears to be required for this condition.

\section{Introduction}

Primary squamous cell carcinoma (SCC) of the thyroid gland is rare, accounting for $<1 \%$ of all thyroid cancers (1). Primary SCC is often diagnosed at a locally advanced stage (2) and has a poor prognosis, with a median overall survival (mOS) of $<6$ months, which is clinically similar to undifferentiated carcinoma of the thyroid gland with an aggressive course (3). While $43 \%$ of undifferentiated carcinomas of the thyroid gland give rise to distant metastases (4), SCC of the thyroid gland has been reported to metastasize in $\sim 25 \%$ of the cases, whereas local invasion is more frequent (5).

Thyroid cancer often metastasizes to the lung and the bone, whereas metastasis to the heart is extremely rare, having been identified in $0-2 \%$ of autopsy cases with thyroid cancer (6). Since the diagnosis of cardiac metastasis of thyroid cancer is difficult due to its asymptomatic nature, only 54 cases have been diagnosed during their lifetime with cardiac metastasis of thyroid cancer over a period of 130 years (7). Although the major histological types of thyroid cancer have been reported to include papillary, follicular and undifferentiated cancer, to the best of our knowledge, no case of SCC of the thyroid gland with cardiac metastasis has been reported to date.

Metastasis of thyroid cancer to the heart may cause various specific symptoms and it has been associated with a poor prognosis. While previous cases mainly resulted in sudden death due to severe arrhythmia and heart failure (7), there has been one report of a coagulation disorder associated with cardiac metastasis of undifferentiated thyroid cancer (8). 
The patient presented herein developed a severe coagulation disorder in association with SCC of the thyroid gland with cardiac metastasis. The coagulation disorder was controlled using chemoradiotherapy for the metastatic lesion of the heart.

\section{Case report}

In April, 2015, a 57-year-old man became aware of a neck mass and consulted the Department of Otolaryngology of the Kyushu University Hospital (Fukuoka, Japan). The patient had no significant past medical history, was a social drinker, had never smoked and had no known allergies. There was no family history of malignant tumors. A computed tomography (CT) scan revealed a thyroid mass invading the trachea and enlargement of the cervical lymph nodes. A fluorodeoxyglucose-positron emission tomography (FDG-PET)/CT scan revealed high-uptake lesions in the thyroid gland and cervical lymph nodes. Fine-needle aspiration biopsy of a cervical lymph node revealed infiltration by papillary thyroid carcinoma (PTC). The patient was diagnosed with PTC, T4aN1bM0, stage IVA according to the 7th edition of the Union for International cancer Control TNM classification of malignant tumors (http://www.uicc. org/sites/main/files/private/TNM_Classification_of_Malignant_ Tumours_Website_15\%20MAy2011.pdf) and underwent total thyroidectomy with bilateral modified neck dissection and tracheotomy. On histological examination, the tumor exhibited papillary carcinoma as well as squamous cell carcinoma (SCC) components (Fig. 1). No anaplastic carcinoma component was evident, which was consistent with SCC of the thyroid gland transformed from PTC. The surgical margin was positive for carcinoma cells, and 8 of the 30 retrieved cervical lymph nodes were positive for metastases; thus, the disease was histologically confirmed as pT4aN1bM0, stage IVA. Due to the positive margin, the patient was administered postoperative chemoradiotherapy consisting of an oral 5-fluorouracil derivative, S-1 ( $80 \mathrm{mg} /$ day), and radiation $(61.4 \mathrm{~Gy} / 33$ fractions) as adjuvant therapy.

Three months after the adjuvant therapy, the patient returned complaining of subcutaneous hemorrhages of the extremities. On admission, the blood pressure was $144 / 81 \mathrm{mmHg}$, the heart rate was $115 / \mathrm{min}$, the temperature was $36.8^{\circ} \mathrm{C}$ and the $\mathrm{SpO}_{2}$ was $96 \%$. The Eastern Cooperative Oncology Group performance status was 2 . Physical examination showed subcutaneous hematomas involving the left thigh and lower leg. There was no thrombocytopenia, but elevation of fibrin/fibrinogen degradation products (FDP) $(332.3 \mu \mathrm{g} / \mathrm{ml})$ and D-dimer $(35.1 \mu \mathrm{g} / \mathrm{ml})$, and consumption of fibrinogen (68 mg/dl) were confirmed on blood examination. The laboratory evaluation was consistent with disseminated intravascular coagulation (DIC). The levels of certain tumor markers, such as carbohydrate antigen $19-9(252.5 \mathrm{U} / \mathrm{ml})$, cytokeratin 19 fragment $(10.8 \mathrm{ng} / \mathrm{ml})$ and SCC antigen $(2.4 \mathrm{ng} / \mathrm{ml})$ were elevated, but those of carcinoembryonic antigen $(1.7 \mathrm{ng} / \mathrm{ml})$ and thyroglobulin $(0.46 \mathrm{ng} / \mathrm{ml})$ were within the normal range. The patient was put on low-molecular-weight heparin therapy (75 IU/kg) and replacement with fresh-frozen plasma. The CT scan revealed no significant findings, apart from the hematomas of the left thigh and lower leg. However, an FDG-PET scan revealed high-uptake lesions in the interventricular septum and left hilar lymph nodes (Fig. 2A). The patient was diagnosed with cardiac and mediastinal lymph node metastases of
SCC of the thyroid gland with DIC. Echocardiography showed preserved systolic function (69.9\%) and a pulmonary arterial wedge pressure of $13.0 \mathrm{mmHg}$. No thrombosis was observed in the cardiac chambers, but a mass sized $52 \mathrm{~mm}$ in greatest diameter was observed protruding towards the right ventricle from the interventricular septum (Fig. 2B). One month after admission, the patient underwent placement of a cardiac pacemaker for the management of complete atrioventricular block caused by cardiac metastasis.

Following pacemaker placement, radiotherapy (41.4 Gy/23 fractions) concurrent with weekly administration of paclitaxel (30 $\mathrm{mg} / \mathrm{m}^{2} /$ week, every 3 weeks) was initiated for the treatment of cardiac and mediastinal lymph node metastases. DIC resolved 18 days after the initiation of chemoradiotherapy, and subcutaneous hemorrhage and leg pain improved significantly. Blood tests showed a normal blood platelet count of $22.3 \times 10^{4} / \mu 1$, fibrinogen of $223 \mathrm{mg} / \mathrm{dl}$, mildly elevated FDP of $10.6 \mu \mathrm{g} / \mathrm{ml}$ and D-dimer of $8.2 \mu \mathrm{g} / \mathrm{ml}$. Although the coagulation disorder was effectively controlled, the size of the cardiac metastasis remained stable.

Approximately 2 weeks after the initiation of chemoradiotherapy, cough and shortness of breath appeared, without fever, respiratory failure, or sputum. The results of the blood tests were as follows: White blood cell count $8,930 / \mu 1$, CRP $10.01 \mathrm{mg} / \mathrm{dl}$, Krebs von den Lungen-6 $300 \mathrm{U} / \mathrm{ml}$, surfactant protein-D $53.3 \mathrm{ng} / \mathrm{ml}$ and procalcitonin $0.28 \mathrm{ng} / \mathrm{ml}$. The results of sputum and blood cultures were negative. CT revealed increased $1-\mathrm{cm}$ nodular shadows spreading in the subpleura of the lungs bilaterally and a pattern of organizing pneumonia (Fig. 3A and B), suggesting the effect of radiation. The bronchoalveolar lavage fluid included $83 \%$ macrophages, $13 \%$ neutrophils and $4 \%$ lymphocytes. Although the increased neutrophil fraction suggested bacterial pneumonia, the CT findings supported radiation pneumonitis and chemoradiotherapy was discontinued at $27 \mathrm{~Gy} / 15$ fractions. Prednisolone $(0.5 \mathrm{mg} / \mathrm{kg})$ was administered with a combination of antibiotics. Despite treatment, respiratory failure progressed and the lung field shadows gradually worsened. Since DIC relapsed following cessation of chemoradiotherapy, systemic therapy for the primary disease was deemed necessary. Lenvatinib ( $24 \mathrm{mg} /$ day) was administered, but no response was observed on echocardiography. Eighteen days after the administration of lenvatinib, the patient's respiratory condition suddenly deteriorated and the nodular shadows expanded to include a wide area of the subpleura (Fig. 3C and D). The DIC progressed further and rapid enlargement of the protruding right ventricular mass was also observed on echocardiography. Twenty-five days after administration of lenvatinib, the patient succumbed to respiratory failure due to alveolar hemorrhage induced by DIC.

\section{Discussion}

Thyroid cancer commonly metastasizes to the lung and bone, while metastasis to the heart is extremely rare. Although cardiac metastasis has been identified in $0-2 \%$ of autopsy cases of thyroid cancer (6), the diagnosis of this type of metastasis during the patient's lifetime is unusual (7). A systematic review of rare metastases of differentiated thyroid cancer, including 42 case series and 197 case reports, demonstrated metastases to the brain, skin and liver, but not to the heart (9). SCC of the 

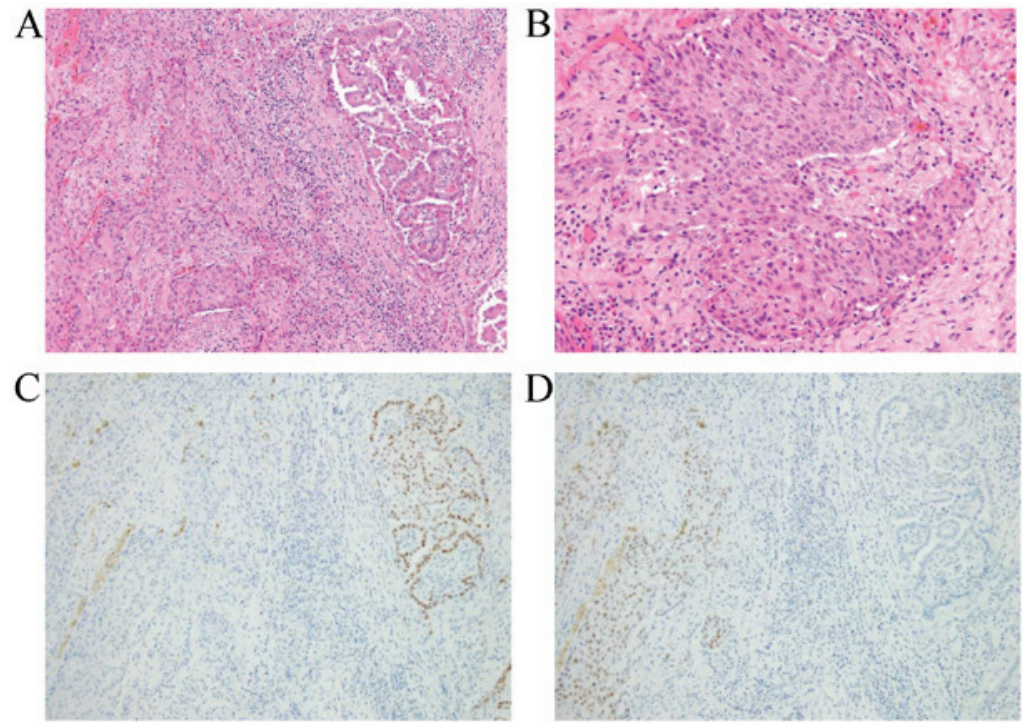

Figure 1. Hematoxylin and eosin staining of the thyroid tumor showing (A) the papillary and squamous cell carcinoma (SCC) components and (B) a higher magnification of the SCC component. The papillary carcinoma and SCC stained positive for (C) thyroid transcription factor-1 and (D) SCC antigen, respectively (magnification: A, C and D, x100; B, x200).
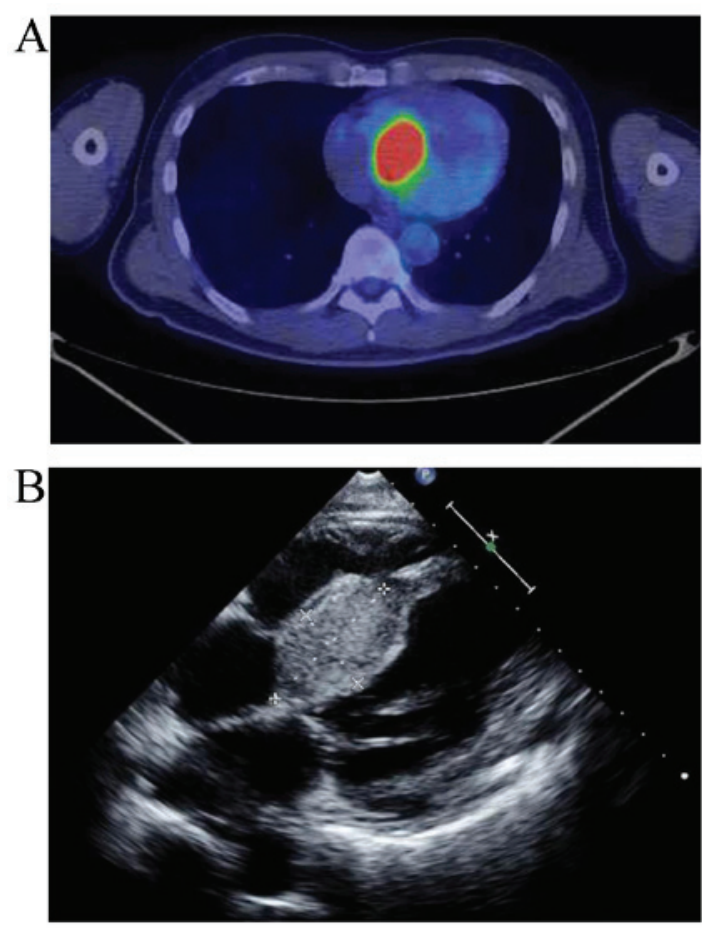

Figure 2. (A) Fluorodeoxyglucose-positron emission tomography scan showing high-uptake lesions in the interventricular septum. (B) Echocardiography showing a mass protruding from the interventricular septum towards the right ventricle.

thyroid gland often progresses rapidly, similar to undifferentiated carcinoma of the thyroid gland, but distant metastases are observed less frequently compared with undifferentiated carcinoma. To the best of our knowledge, the present study is the first report of SCC of the thyroid gland with cardiac metastasis.

Carcinoma of the thyroid gland metastasizing to the right and left ventricles and other parts of the heart has been reported $(7,8)$, with cardiac symptoms including arrhythmia,
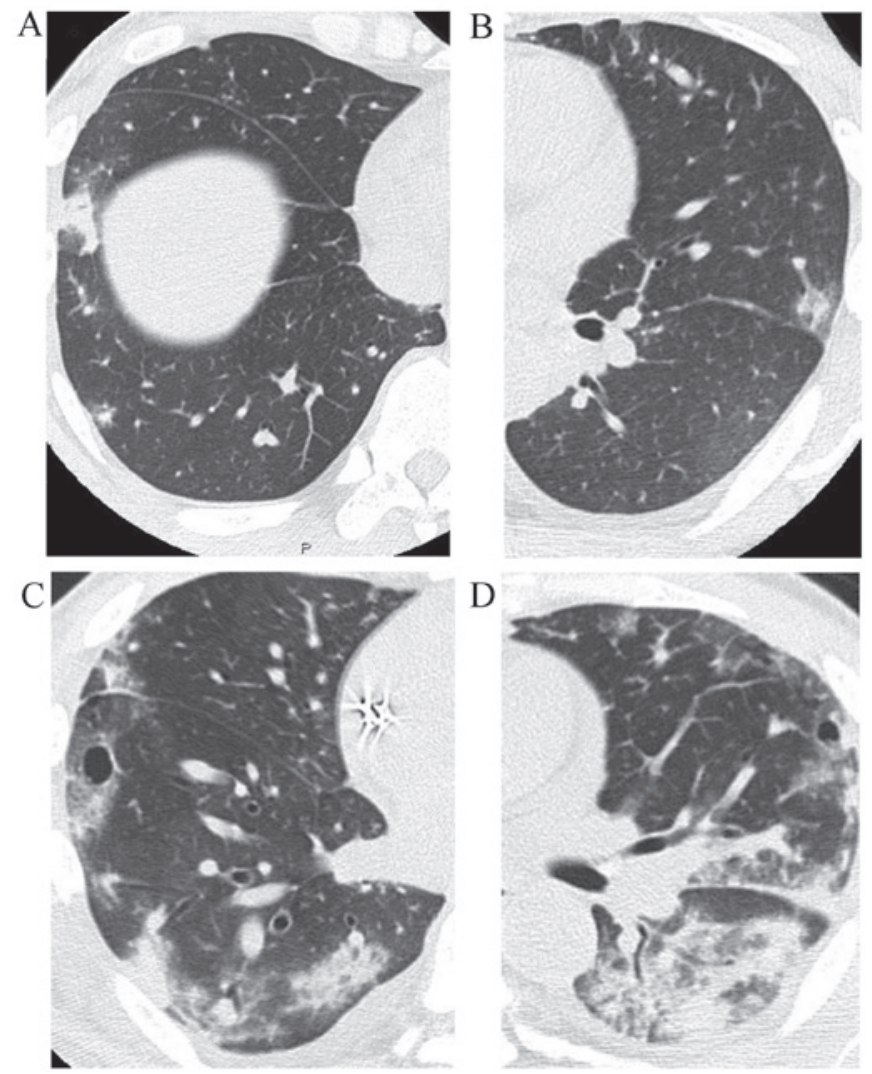

Figure 3. (A and B) Computed tomography scan showing 1-cm nodular shadows spreading in the subpleura of the lungs bilaterally. (C and D) Eighteen days after administration of lenvatinib, the shadows had expanded to involve a wider area of the subpleura.

heart failure and valvular disease. Since surgical intervention for the tumor may achieve a good prognosis, it should be considered; however, there are few such cases. The prognosis of cardiac metastasis has not been fully elucidated, but is expected to be poor based on the frequent cases of sudden 
death from heart complications (7). Our patient also suffered from impaired atrioventricular conduction, requiring insertion of a pacemaker. Although the tumor was located in the interventricular septum and protruded into the right ventricle, there was no obvious right heart failure.

Severe DIC in a patient with a solid malignant tumor is relatively rare. A prospective study by Sallah et al demonstrated that 76 of 1,117 patients $(6.8 \%)$ with solid tumors had DIC (10). A multivariate analysis in that study revealed that old age, breast cancer and necrosis in the tumor tissue were risk factors for DIC (10). Additionally, high tumor burden with multiple metastases and bone marrow metastasis of gastric or breast cancer are often associated with DIC. Although a case of DIC in association with cardiac metastasis from thyroid cancer was previously reported, this finding is quite rare (8).

DIC in patients with solid tumors is mainly caused by tissue factor production by the tumor cells and increasing tissue factor accumulation on the surface of monocytes and macrophages. Activation of the extrinsic pathway by excessive tissue factors induces DIC, and direct exposure of the tumor surface to the plasma may also contribute to induction of DIC (11). A metastatic cardiac tumor protruding into the ventricle may cause severe DIC, as reported in a previous such case (12). The metastatic tumor in the right ventricle is also considered to have been the cause of the severe DIC in the present case.

Control of tumor-associated DIC requires not only supportive care, but also antitumor therapy. A retrospective analysis of the treatment of DIC in association with advanced gastric or colorectal cancer demonstrated that the patient group treated with chemotherapy was superior in terms of overall survival and amelioration of symptoms caused by DIC to the group receiving best supportive care (13). Although one of the standard treatments for metastatic thyroid cancer is chemotherapy with kinase inhibitors (14-16), it was not possible in the present case due to the clinical bleeding tendency. Weekly administration of paclitaxel $\left(30 \mathrm{mg} / \mathrm{m}^{2}\right)$ was applied based on its survival benefit and safety profile (17) for anaplastic thyroid cancer and consideration of its safety with concomitant external beam radiation (18). Intensive supportive care did not improve DIC in our patient, but irradiation of the metastases to the heart and the hilar lymph nodes in combination with systemic chemotherapy with paclitaxel resulted in significant improvement.

Lung metastasis appears in 7-12\% of thyroid cancer patients (19). Multiple nodular or granular shadows sized $0.5-3 \mathrm{~cm}$ are generally observed on radiographic examination (19). Although no lung metastases were detected at the time of diagnosis of the cardiac metastasis by CT and PET examinations in the present case, diffuse irregular infiltrative shadows, sized $1 \mathrm{~cm}$, appeared in both peripheral lung fields after the initiation of chemoradiotherapy. Bacterial, viral, or fungal infections were not considered likely based on the symptoms and the results of the cultures and laboratory tests. Steroid treatment was administered for possible irradiation pneumonia, but was ineffective. Thyroid cancer has been recognized as one of the diseases commonly associated with pulmonary tumor embolism (20-22). Luo et al reported pulmonary tumor embolism in a patient with right ventricular metastasis of thyroid cancer based on the facts that the patient had no deep venous thrombosis and was on anticoagulant therapy (23). Although histological diagnosis was not available, the presence of a tumor protruding into the right ventricle and worsening of the condition even with anticoagulant therapy suggested pulmonary tumor embolism leading to acute respiratory distress syndrome in our patient.

The aim of this study was to present an extremely rare case of cardiac metastasis of SCC of the thyroid gland complicated by a severe coagulation disorder. Intensive local control of the metastatic tumor by concurrent chemoradiotherapy is required to control the coagulation disorder in such cases and, in case of unexplained DIC, cardiac metastasis should be considered.

\section{References}

1. Zhou XH: Primary squamous cell carcinoma of the thyroid. Eur J Surg Oncol 28: 42-45, 2002.

2. Syed MI, Stewart M, Syed S, Dahill S, Adams C, McLellan DR and Clark LJ: Squamous cell carcinoma of the thyroid gland: Primary or secondary disease? J Laryngol Otol 125: 3-9, 2011.

3. Lam KY, Lo CY and Liu MC: Primary squamous cell carcinoma of the thyroid gland: An entity with aggressive clinical behaviour and distinctive cytokeratin expression profiles. Histopathology 39: 279-286, 2001.

4. Kebebew E, Greenspan FS, Clark OH, Woeber KA and McMillan A: Anaplastic thyroid carcinoma. Treatment outcome and prognostic factors. Cancer 103: 1330-1335, 2005.

5. Zhang YX, Zhang B, Wu YH, Liu WS, Liu SY, Gao L, Xu ZG and Tang PZ: Primary squamous cell carcinoma of the thyroid: Retrospective analysis of 28 cases. Zhonghua Er Bi Yan Hou Tou Jing Wai Ke Za Zhi 48: 143-147, 2013 (In Chinese).

6. Abraham KP, Reddy V and Gattuso P: Neoplasms metastatic to the heart: Review of 3314 consecutive autopsies. Am J Cardiovasc Pathol 3: 195-198, 1990.

7. Catford SR, Lee KT, Pace MD, Marasco SF, Longano A and Topliss DJ: Cardiac metastasis from thyroid carcinoma. Thyroid 21: 855-866, 2011.

8. Hara K, Ohno M, Takenaga M, Tsuneyoshi H, Takeuchi H, Kashida M, Yamaguchi T, Machii K, Furuta S and Tohda E: Metastatic thyroid cancer to the right ventricle causing obstruction of the right ventricular outflow tract and associated with disseminated intravascular coagulopathy: A case report. J Cardiogr 16: 765-767, 1986 (In Japanese).

9. Madani A, Jozaghi Y, Tabah R, How J and Mitmaker E: Rare metastases of well-differentiated thyroid cancers: A systematic review. Ann Surg Oncol 22: 460-466, 2015.

10. Sallah S, Wan JY, Nguyen NP, Hanrahan LR and Sigounas G: Disseminated intravascular coagulation in solid tumors: Clinical and pathologic study. Thromb Haemost 86: 823-833, 2001.

11. Sutherland DE, Weitz IC and Liebman HA: Thromboembolic complications of cancer: Epidemiology, pathogenesis, diagnosis, and treatment. Am J Hematol 72: 43-52, 2003.

12. John $\mathrm{T}$ and Davis ID: Ventricular metastasis resulting in disseminated intravascular coagulation. World J Surg Oncol 24: $29,2005$.

13. Feinstein DI: Disseminated intravascular coagulation in patients with solid tumors. Oncology (Williston Park) 29: 96-102, 2015.

14. Wells SA Jr, Robinson BG, Gagel RF, Dralle H, Fagin JA, Santoro M, Baudin E, Elisei R, Jarzab B, Vasselli JR, et al: Vandetanib in patients with locally advanced or metastatic medullary thyroid cancer: A randomized, double-blind phase III trial. J Clin Oncol 30: 134-141, 2012.

15. Brose MS, Nutting CM, Jarzab B, Elisei R, Siena S, Bastholt L, de la Fouchardiere C, Pacini F, Paschke R, Shong YK, et al: Sorafenib in radioactive iodine-refractory, locally advanced or metastatic differentiated thyroid cancer: A randomized, double-blind, phase 3 trial. Lancet 384: 319-328, 2014.

16. Schlumberger M, Tahara M, Wirth LJ, Robinson B, Brose MS, Elisei R, Habra MA, Newbold K, Shah MH, Hoff AO, et al: Lenvatinib versus placebo in radioiodine-refractory thyroid cancer. N Engl J Med 372: 621-630, 2015.

17. Higashiyama T, Ito Y, Hirokawa M, Fukushima M, Uruno T, Miya A, Matsuzuka F and Miyauchi A: Induction chemotherapy with weekly paclitaxel administration for anaplastic thyroid carcinoma. Thyroid 20: 7-14, 2010. 
18. Onoda N, Sugitani I, Higashiyama T, Hara H, Ito K, Kammori M, Sugino K, Suzuki S, Toda K, Yoshida A and Miyauchi A: Concept and design of a nationwide prospective feasibility/efficacy/safety study of weekly paclitaxel for patients with pathologically confirmed anaplastic thyroid cancer (ATCCJ-PTX-P2). BMC Cancer 15: 475, 2015.

19. Fraser RS, Muller NL, Colman N, et al: Pulmonary neoplasms. Diagnosis of disease of the chest. 4th edition. Philadelphia, W.B. Saunders Company, pp1405-1407, 1999.

20. Bassiri AG, Haghighi B, Doyle RL, Berry GJ and Rizk NW: Pulmonary tumor embolism. Am J Respir Crit Care Med 155: 2089-2095, 1997.
21. Chan CK, Hutcheon MA, Hyland RH, Smith GJ, Patterson BJ and Matthay RA: Pulmonary tumor embolism: A critical review of clinical, imaging, and hemodynamic features. J Thorac Imaging 2: 4-14, 1987

22. Roberts KE, Hamele-Bena D, Saqi A, Stein CA and Cole RP Pulmonary tumor embolism: A review of the literature. Am J Med 115: 228-232, 2003.

23. Luo H, Tulpule S, Alam M, Patel R, Sen S and Yousif A: A rare silent killer: Right atrial metastasis of thyroid hürthle cell carcinoma. Case Rep Oncol 8: 233-237, 2015. 